
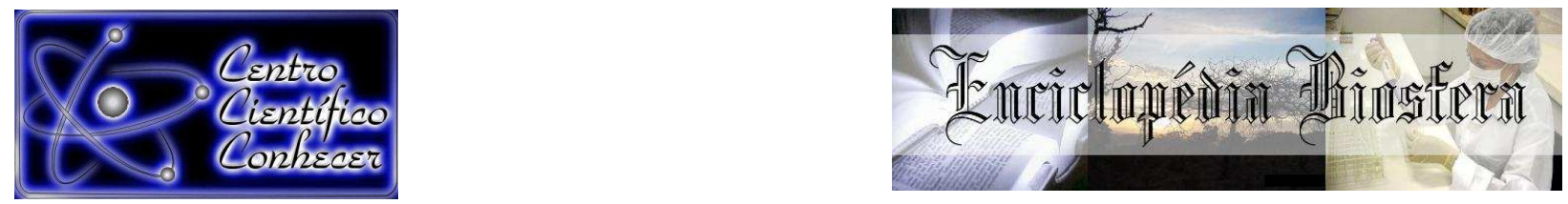

\title{
ESTRUTURA E DISTRIBUIÇÃO ESPACIAL DE Calophyllum brasiliense Cambess. (CALOPHYLLACEAE) EM FLORESTA INUNDÁVEL DO VALE DO GUAPORÉ, AMAZÔNIA MATO-GROSSENSE
}

Michele de Morais ${ }^{1}$ Solange Kimie Ikeda Castrillon ${ }^{2}$ Carolina Joana da Silva ${ }^{2,3}$ Célia Regina Araújo Soares-Lopes ${ }^{2}$ Nilo Leal Sander ${ }^{3}$

1. Graduação em Ciências Biológicas pela Universidade do Estado do Mato Grosso, Campus de Cáceres - Mato Grosso, Brasil. e-mail: michele brasnorte@hotmail.com.

2. Docente do Curso de Ciências Biológicas - UNEMAT, Campus de Cáceres e Alta Floresta.

3. Doutorado em Biodiversidade e Biotecnologia da Amazônia Legal, Rede Bionorte.

Recebido em: 08/09/2015 - Aprovado em: 14/11/2015 - Publicado em: 01/12/2015 DOI: http://dx.doi.org/10.18677/Enciclopedia_Biosfera_2015_017

A capacidade de adaptação fisiológica de uma espécie em locais de maior saturação hídrica contribui para a seletividade destas permitindo que algumas tenham vantagens sobre outras, a exemplo tem-se Calophyllum brasiliense Cambess (Calophyllaceae), que é capaz de se desenvolver em áreas alagáveis. Devido a tais características o presente estudo teve por objetivo realizar uma análise da distribuição espacial do guanandi ( $C$. brasiliense) em áreas com pulso de inundação anual no Rio Guaporé, Município de Vila Bela da Santíssima Trindade - MT. As análises basearam-se em coletas de dados fitossociológicos em 1 ha, essa área foi dividida em 10 transectos de 10×100m (subdivididos em $10 \mathrm{~m}$ parcelas de $10 \times 10 \mathrm{~m}$ cada) perpendiculares ao leito do rio, CAP (Circunferência à Altura do Peito, 1,30 m do solo) maior ou igual a $15 \mathrm{~cm}$. A densidade da espécie no rio Guaporé foi de 43 ind.ha ${ }^{-1}$. A distribuição espacial apresentou um padrão aleatório com valores de Imst $(0,49)$ com maior numero de indivíduos nas menores classes de tamanho, representando uma tendência de $\mathrm{J}$ invertido. As médias de CAP dos indivíduos estatisticamente não se diferem significativamente. A espécie encontra-se em um processo de regeneração, este resultado pode ser observado devido ao numero de indivíduos encontrados nas menores classes de tamanho. Tais fatores podem estar relacionados a ações antrópicas, observadas na área de estudo.

PALAVRAS-CHAVE: áreas úmidas, regeneração, saturação hídrica.

\section{STRUCTURE AND SPATIAL DISTRIBUTION OF Calophyllum brasiliense Cambess.(CALOPHYLLACEAE) IN FLOODED THE GUAPORÉ VALLEY FOREST, AMAZON MATO-GROSSENSE}

\begin{abstract}
The physiological adaptive capacity of some species in higher water saturation sites contributes to the selectivity of them, allowing some advantages over others ones, such as Calophyllum brasiliense Cambess (Calophyllaceae), which is able to develop in areas wetland. Because of these characteristics, the present study aimed to carry out an analysis of the spatial distribution of guanandi (C. brasiliense) in areas with an
\end{abstract}


annual flood pulse in the Guaporé River, at Vila Bela da SantíssimaTrindade - MT, which could contribute to conservation and recovery. The analyzes are based on phytosociological data collection and flora in 1 ha, this area had 10 transects $10 \times 100 \mathrm{~m}$ (subdivided into 10 plots of $10 \times 10 \mathrm{~m}$ each) perpendicular to the riverbed, $\mathrm{DBH}$ (Diameter at breast height, 1,30m height) greater than or equal to $15 \mathrm{~cm}$. The density of the species in the Guaporé River was 43 ind.ha $^{-1}$. The spatial distribution showed a random pattern with Imst (Morisita Index) values (0.49) with the highest number of individuals in the smaller size classes, representing an inverted $\mathrm{J}$ trend. The average DBH individuals were not found statistically significant. The species are in regeneration process; observe this result due to the number of individuals found in the smaller size classes. These factors relate to anthropic actions observed in the study area.

KEYWORDS: wetlands, regeneration, watersaturation.

\section{INTRODUÇÃO}

A biodiversidade brasileira é considerada patrimônio natural devido ao vasto território, à diversidade e ao endemismo de espécies, que caracterizam uma heterogeneidade nas formações ecossistêmicas de seus biomas, apresentando assim grande importância acerca da conservação da biodiversidade global (ASSUNÇÃO \& FELFILI, 2004). O Estado de Mato Grosso no seu amplo espaço territorial de 903.378.292 $\mathrm{Km}^{2}$ (IBGE, 2015), contempla três grandes biomas brasileiros, Amazônia, Cerrado e Pantanal, formando um mosaico de ambientes naturais que se diferenciam tanto pela estrutura, como pelas espécies e densidade de indivíduos por hectare, ostentando uma situação privilegiada, com grande potencial de recursos naturais (FACHIM \& GUARIM, 1995). Destaca - se nesses biomas, extensivas áreas úmidas de diferentes tipos, as quais segundo ARIEIRA e CUNHA (2006), cobrem no mundo cerca de 250 milhões de hectares com grande importância nos ciclos biogeoquímicos e como habitats para espécies.

As áreas úmidas em suas oscilações de nível da água têm sua descrição e estrutura caracterizadas pelo conceito de pulso de inundação, pois este define a relação de água, organismo e nutrientes entre rios e lagos com áreas alagáveis. Esta relação define a assinatura do padrão hidrológico, influenciando nas alterações ecológicas entre os ambientes, aquático e terrestre (JUNK et al., 1989).

Além do Pantanal Mato-Grossense, considerada a maior área úmida do planeta (JUNK \& DA SILVA, 1999), o estado de Mato Grosso abarca aquelas típicas do bioma Cerrado, como as veredas, com seus expressivos buritizais e as do bioma amazônico, tipificadas como a maior área de florestas inundáveis do mundo, as quais abrangem cerca de $350.000 \mathrm{~km}^{2}$, correspondente a $6 \%$ da Amazônia brasileira (MELACK \& HESS, 2010; JUNK et al., 2011 ).

Nas áreas úmidas localizadas às margens de rios inundáveis, no bioma Cerrado, ocorrem os tipos de vegetação arbórea adaptada à beira de rios tais como: floresta de galeria, várzea ripária, entre outras, as quais proporcionam ao local, a formação de mosaicos determinados por condições de solo, altitude, clima e regime hídrico dos rios e cursos d'água (BELLUTA, 2012). A vegetação em ambientes inundáveis possui uma ampla dominância e tem o predomínio de poucas espécies conspícuas, sendo de fundamental importância no que diz respeito à sua contribuição na contenção de sedimentos, regularização de vazões, erosão de margens, e proteção da fauna aquática (PEREIRA \& PEREIRA, 2010).

A capacidade de adaptação fisiológica de uma espécie em locais de saturação hídrica contribui para a seletividade das espécies permitindo que uma tenha 
vantagem sobre outras (SARMENTO et al., 2013), a exemplo tem-se a Calophyllum brasiliense Cambess (guanandi). pertencente à família Calophyllaceae, que é capaz de crescer dentro da água e em áreas de mangue (LORENZI, 1992). Segundo MARQUES (1994), o C. brasiliense é uma espécie extremamente tolerante à saturação hídrica do solo, que vai desde solos úmidos e periodicamente inundáveis até solos permanentemente encharcados e brejosos, podendo permanecer nestas condições por um longo período. Neste último caso, $C$. brasiliense, muitas vezes tem vantagem seletiva sobre outras espécies e passa a ocorrer em destaque nos parâmetros quantitativos (IVANAUSKAS, 1997).

Indivíduos de $C$. brasiliense têm sua distribuição espacial influenciada pelas condições edáficas do ambiente, mesmo tolerantes ao alagamento do solo apresentam o crescimento mais lento em comparação a outras espécies, e em condições de solo saturado seu crescimento se torna ainda mais lento (DEVIDE et al., 2011). Com ocorrência desde a América Central até a costa Sudeste do Brasil, o guanandi ( $C$. brasiliense) é uma das espécies arbórea mais importante em termos de dominância relativa, pois está presente na Floresta Amazônica, Mata Atlântica e Pantanal (GUARIM-NETO et al., 2010, p. 1) incluindo restingas e planícies costeiras dessa região, e nas florestas pantanosas se formam nas depressões dos Cerrados (OLIVEIRA \& JOLY, 2010), é considerada climácica por apresentar regeneração abundante na sombra. A espécie floresce nos meses de outubro a novembro, e frutifica por um período de 10 meses, sendo considerada uma espécie que está em expansão em matas que não sofrem pressão antrópica (MARQUES \& JOLY, 2000). No Brasil tem alto valor econômico, devido a sua larga utilização para os mais diversos fins, tais como: construção civil, marcenaria, construção naval (LORENZI, 1992).

Estudos florísticos e fitossociológicos de uma área possibilitam análises estruturais comparativas com o mesmo conjunto de espécies, se há dominância em um local ou, uma distribuição de abundâncias de maior similaridade entre as espécies (MORO \& MARTINS, 2011). Tais variações podem ser explicadas pela regeneração natural, a qual segundo REIS et al. (2009) atua dentro das populações em relação à adaptação ao ambiente aliada a fatores demográficos e ecológicos. De acordo com GAMA et al. (2003) as fases de regenerações contribuem para o estabelecimento e desenvolvimento das plantas, possibilitando a preservação, conservação e formação de novas florestas. Na perspectiva de contribuir com o conhecimento desses processos de capacidade de adaptação, regeneração e distribuição dessa espécie em uma floresta aluvial, este trabalho tem por objetivo realizar uma análise da distribuição espacial do guanandi (Calophyllum brasiliense) em áreas com inundação, no Rio Guaporé, Município de Vila Bela da Santíssima Trindade - MT.

\section{MATERIAL E MÉTODOS}

\section{Caracterização da área de estudo}

A área de estudo está localizada em uma mata ciliar inundável do Rio Guaporé com pulso de inundação anual de longa duração e de média amplitude $(5 \mathrm{~m})$, situada no Vale do Alto Guaporé, no município de Vila Bela da Santíssima Trindade, entre as coordenadas 15 $03^{\prime} 56.16^{\prime \prime}$ S e 59 $52^{\prime} 27.36^{\prime \prime}$ O, com elevação de $203 \mathrm{~m}$ e $15^{\circ} 02^{\prime} 29.14^{\prime \prime} \mathrm{S}$ e 595' $32.92^{\prime \prime}$ O, e $197 \mathrm{~m}$ ao nível do mar. 
A região do Vale do Guaporé está localizada na Região Sudeste da Bacia Amazônica e Norte do Pantanal Mato-grossense, inserida em dois domínios morfoclimáticos, Floresta Amazônica e Cerrado (AB'SABER,1967).

O clima é do tipo tropical úmido ou subúmido (AB'SABER, 1967; MOSS \& MOSS, 2007) com temperatura média de 24ํ $\mathrm{C}$ e 26으 (KOEPPEN, 1948), é caracterizado por três a quatro meses secos (de junho a setembro) com chuvas de verão e precipitação anual média de $2150 \mathrm{~mm}$ (BRASIL, 1979). Tem como principal rio responsável por sua drenagem, o Rio Guaporé, que ocupa uma área de aproximadamente $38.563,81 \mathrm{Km}^{2}$ (BRASIL - MMA, 2006), com cerca de $1.150 \mathrm{~km}$ de extensão navegável. Compreende uma região com grandes extensões de "várzeas" inundadas nos períodos de cheia (MAITELLI, 2005), presente nos municípios de Vila Bela da Santíssima Trindade, Pontes e Lacerda, Comodoro e Nova Lacerda, abrigando uma enorme variedade de ambientes (FOSCHIERA \& MIORIM, 2006). Predominam na região, solos do tipo Latossolos, Argissolos e Neossolos quartzarênicos, nas áreas mais elevadas se encontram Gleissolos e Plintossolos nas áreas mais baixas sujeitas ao alagamento pluvial, devido à drenagem deficiente (PIERANGELI et al., 2009).

\section{Procedimentos em campo}

Para a análise da estrutura populacional de Calophyllum brasiliense Cambess. foram instaladas parcelas permanentes dividida em 10 transectos de 10x100 (subdivididos em 10 parcelas de 10x10 cada) adaptado por FELFILI (2005) (Figura 1), perfazendo 1 ha, traçadas perpendicularmente ao leito do Rio Guaporé. Nestas parcelas foram mensurados todos os indivíduos com CAP (circunferência à altura do peito, $1,30 \mathrm{~m}$ do solo) maior ou igual a $15 \mathrm{~cm}$. A altura de cada indivíduo foi estimada visualmente.
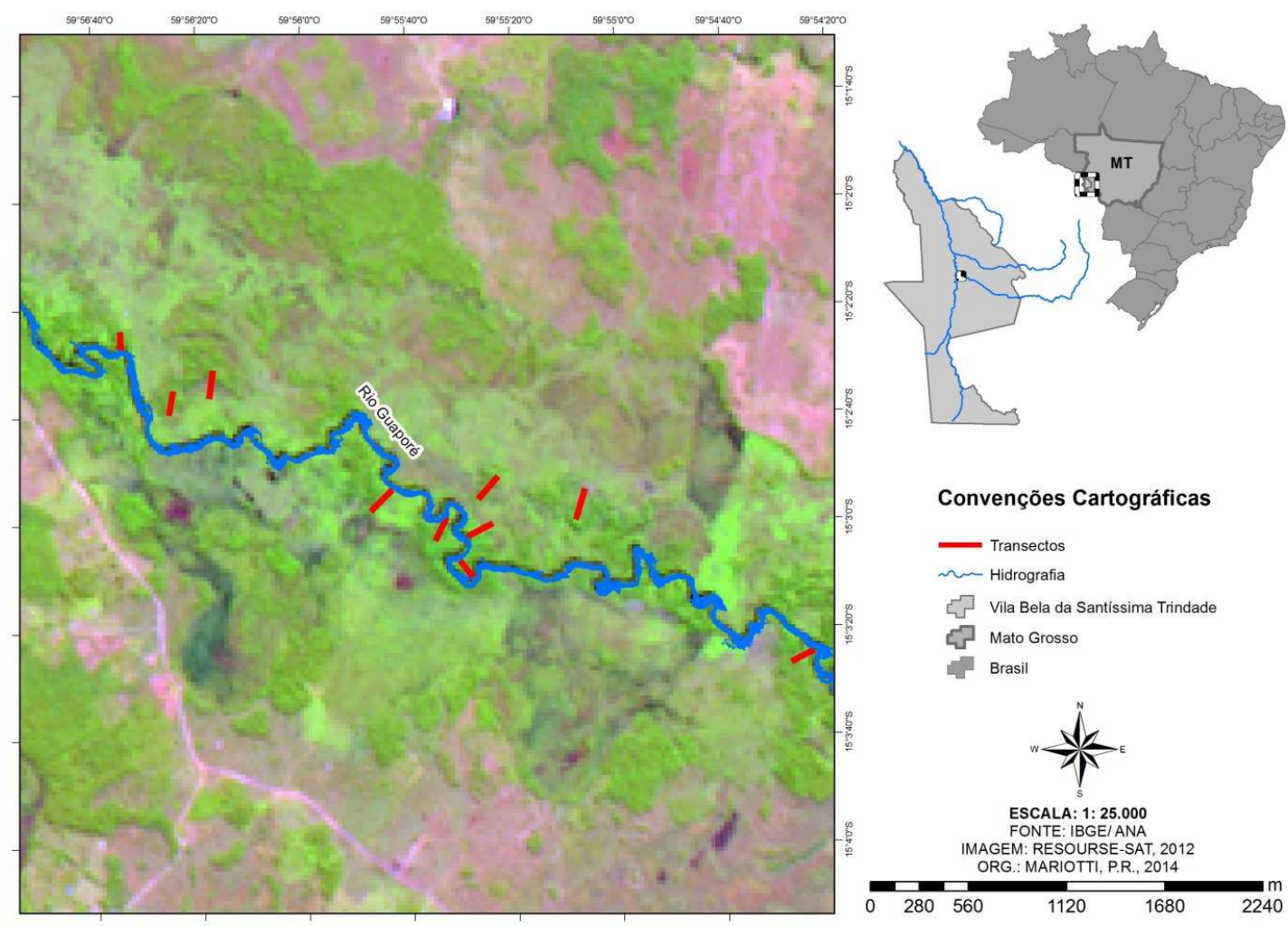

Convenções Cartográficas

Transectos

m Hidrografia

5 Vila Bela da Santissima Trindade

Mato Grosso

Brasil

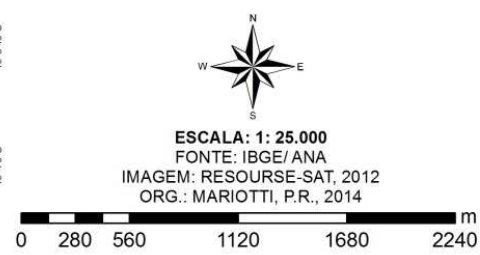

FIGURA 1. Localização da área de estudo ao longo do Rio Guaporé. 


\section{Análise dos dados}

O número total de indivíduos amostrados foi dividido pelo tamanho da área estudada para a obtenção da densidade (ind. ha ${ }^{-1}$ ). Os intervalos de classes para a confecção dos histogramas de altura e diâmetro foram obtidos utilizando-se a fórmula: $\mathrm{A} / \mathrm{K}$, em que $\mathrm{A}$ representa a amplitude dos valores de altura e $\mathrm{K}$ indica uma constante definida pelo algoritmo de Sturges, que consiste em $1+3,3 \times \log 10 \mathrm{n}$, em que $n$ é o número total de indivíduos amostrados (BONINI \& BONINI, 1972).

O resultado dessa razão $(\mathrm{A} / \mathrm{K})$ representa o valor do incremento empregado para definir os intervalos de classes. Para a determinação da distribuição espacial dos indivíduos dentro da área de estudo, foi adotado o índice de Morisita (MORISITA, 1959):

$$
I_{d}=\frac{n \cdot\left(\sum_{i=1}^{s} X^{2}-N\right)}{N \cdot(N-1)}
$$

Em que: Id: índice de Morisita; $\mathrm{n}$ : número total de parcelas amostradas; $\mathrm{N}$ : número total de indivíduos por espécies, contidos em $n$ parcelas; $X^{2}$ : quadrado do número dos indivíduos por parcela; s: número de espécies amostradas.

A significância dos valores calculados para o índice de Morisita (Id) foi obtida mediante 0 teste do qui-quadrado a um nível de significância de 0,05 de probabilidade de erro.

$$
X^{2}=\frac{n \cdot \sum_{i=1}^{s} X^{2}}{N}-N
$$

Para constatar a hipótese nula da probabilidade aleatória utilizaram-se os valores inferior e superior $(0,025$ e 0,975$)$ da distribuição do $x^{2}$ com n-1 graus de liberdade, à significância de 0.05 . Valor de $p<0,025$ ou $p>0,975$ rejeita-se a hipótese de nulidade. Foi também avaliado o Índice de Uniformidade - MUNI que se refere à medida da distribuição no número de indivíduos entre espécies, e o Índice de Agregação - MCLU que indica a distribuição espacial das espécies (HAIRSTON et al., 1971; KREBS, 1999).

Onde: limites superiores e inferiores do Índice de Morisita indicam uma distribuição aleatória. Se IMOR $>M C L U$, a espécie tem uma distribuição espacial agregada. Se IMOR<MUNI, o critério de distribuição espacial é regular. Para IMST, valores entre $-0,5$ a 0,5 indicam uma distribuição aleatória. Valores inferiores a -0,5 indicam uma distribuição regular e valores acima de 0,5 indica distribuição agregada. (KREBS, 1989 citado por PAULA \& SOARES, 2011).

Os dados com as médias das medidas de CAP por transecto foram testados e transformados em valores logarítmicos. Foi feita análise de variância ANOVA (Tukey $\alpha=0,05)$, e plotado em gráfico tipo boxplot. Todas as análises foram realizadas com auxílio da interface estatística $R$.

\section{RESULTADOS E DISCUSSÃO}

A densidade de $C$. brasiliense registrada foi de 43 ind.ha ${ }^{-1}$ distribuídos em 09 dos 10 transectos. A densidade de uma população pode demonstrar a sua forma de regeneração do passado e presente (AGREN \& ZACKRISSON, 1990). MARQUES \& 
JOLY (2000) registraram131 indivíduos em um fragmento de floresta higrófila localizado na bacia do rio Jacaré-Pepira, em Brotas - SP, em uma área de 13 ha dividida em 144 parcelas. Já SCHIAVINI (1992) em uma mata de galeria, paralela ao ribeirão do Panga na estação Ecológica do Panga- MG descreveu em três ambientes distintos de acordo com o nível de inundação, em uma área de 01 ha das duas áreas de seu estudo, a ocorrência de 359 indivíduos, onde no primeiro ambiente - dique, faixa estreita de $10 \mathrm{~m}$ mais próxima ao rio, caracterizada como uma zona de deposição de sedimentos, portanto mais elevada e não sujeita a inundações sazonais. Já no segundo ambiente - meio da mata, depressão localizada após o dique, com solo hidromórfico e saturado de água na maior parte do ano, e no terceiro ambiente - borda da mata, também com $10 \mathrm{~m}$ de largura delimitados, se caracteriza por fazer limite com a vegetação campestre apresentaram indivíduos das maiores classes de tamanho.

Para JANZEN (1970), em florestas tropicais, dois processos são responsáveis por gerar comunidades arbóreas com variabilidade estrutural, a baixa densidade das espécies e a distribuição regular dos adultos; afirma ainda que em uma área, independente da forma de distribuição, a propagação de qualquer espécie declina com a distância das árvores parentais.

No histograma de altura e diâmetro, verificou-se a predominância de indivíduos nas menores classes, este padrão nos indica a capacidade regenerativa da espécie na área de estudo ( $R^{2} 0,659$ para diâmetro e $R^{2} 0,799$ para altura), que pode graficamente ser representado por uma curva do tipo de $\mathrm{J}$ invertido (Figura $2 \mathrm{a}$ e2b). MARQUES \& JOLY (2000) relataram em seu estudo que a distribuição para as classes de tamanhos dos indivíduos predominou nas plantas com classes menores, apresentando progressiva queda nas classes maiores (Quadro 1). Tais resultados podem ter sido constatados devido aos diferentes micro habitats do leito dos rios já que, assim como observado no presente estudo, os locais de estudo apresentam um mosaico de áreas inundadas no período chuvoso, e drenadas no período de estiagem, que se alternam durante o ano. No período chuvoso, os indivíduos são impactados pelo efeito do carreamento da necromassa ou da serrapilheira e do solo, favorecendo as classes posteriores e indicando que está havendo regeneração da espécie.
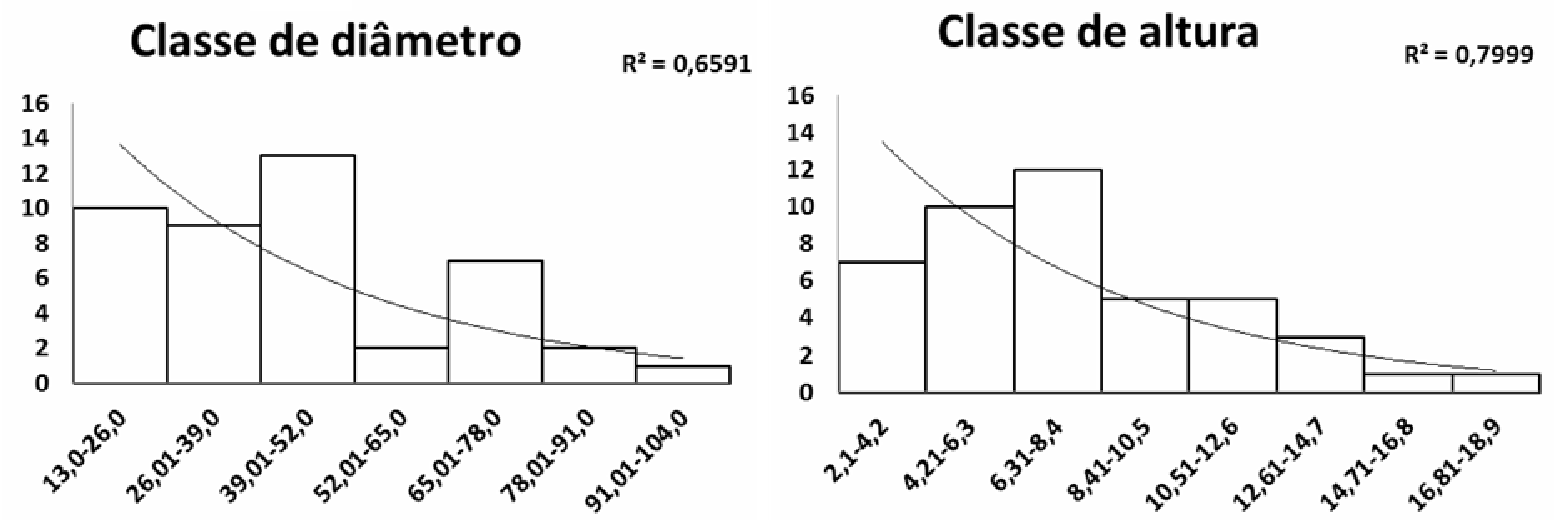

FIGURA 2. Distribuição da circunferência (Circunferência a Altura do Peito - CAP) (a) e alturas (b) dos indivíduos de uma população de Calophyllum brasiliense Cambess. em uma mata ciliar no Rio Guaporé, situada no Vale do Alto Guaporé, município de Vila Bela da Santíssima Trindade, Mato Grosso. 
QUADRO 1. Ocorrência de populações de guanandi $C$. brasiliense em biomas com diferentes condições ambientais.

\begin{tabular}{|c|c|c|c|c|c|c|c|c|c|c|}
\hline \multirow{2}{*}{ Bioma } & \multirow{2}{*}{ Rio } & \multirow{2}{*}{ Ambiente } & \multirow{2}{*}{$\begin{array}{l}\text { Densidade/ } \\
\text { diâmetro }\end{array}$} & \multicolumn{3}{|c|}{ Altura máxima e média } & \multirow{2}{*}{ Tipo J } & \multirow{2}{*}{ Distribuição } & \multirow{2}{*}{$\begin{array}{l}\text { Id (Indice } \\
\text { de Morisita) }\end{array}$} & \multirow{2}{*}{ Fonte } \\
\hline & & & & $\operatorname{Max}$ & Med & Min & & & & \\
\hline Mata Atlântica & $\begin{array}{c}\text { Rio } \\
\text { Jacaré- } \\
\text { Pepira }\end{array}$ & Floresta higrófila & 0,025 & $10 \mathrm{~m}$ & $2 m$ & $0,2 m$ & - & Agregada & $1,46-1,81$ & $\begin{array}{l}\text { MARQUES } \\
\& \text { JOLY } \\
2000\end{array}$ \\
\hline \multirow{3}{*}{ Cerrado } & \multirow[b]{3}{*}{$\begin{array}{l}\text { Estação } \\
\text { Ecológica } \\
\text { do Panga }\end{array}$} & $\begin{array}{l}\text { Dique (não } \\
\text { sujeita a } \\
\text { inundação } \\
\text { periódica) }\end{array}$ & \multirow[b]{3}{*}{$5,0-9,9 \mathrm{~cm}$} & $8 m$ & $5 m$ & $2 m$ & \multirow[b]{3}{*}{ - } & & - & \multirow{3}{*}{$\begin{array}{c}\text { SCHIAVINI } \\
(1992)\end{array}$} \\
\hline & & $\begin{array}{c}\text { Meio (depressão } \\
\text { inundada com } \\
\text { solo } \\
\text { hidromórfico) }\end{array}$ & & $10 \mathrm{~m}$ & $8 m$ & $1 \mathrm{~m}$ & & & - & \\
\hline & & $\begin{array}{c}\text { Borda (limite } \\
\text { Mata de Galeria } \\
\text { - faixa estreita de } \\
\text { campo úmido) }\end{array}$ & & $6 m$ & $3 m$ & $0,5 \mathrm{~m}$ & & & - & \\
\hline $\begin{array}{l}\text { Transição Floresta } \\
\text { Amazônica/Cerrado }\end{array}$ & $\begin{array}{c}\text { Rio } \\
\text { Guaporé }\end{array}$ & $\begin{array}{l}\text { Floresta } \\
\text { periodicamente } \\
\text { inundada }\end{array}$ & 0,659 & $18,9 \mathrm{~m}$ & $10,5 \mathrm{~m}$ & $2,1 \mathrm{~m}$ & Invertido & Aleatória & 0,49 & $\begin{array}{l}\text { Este } \\
\text { estudo }\end{array}$ \\
\hline \multirow{3}{*}{$\begin{array}{c}\text { Transição } \\
\text { Cerrado/Floresta } \\
\text { Amazônica }\end{array}$} & \multirow[b]{3}{*}{ - } & - & & \multirow[b]{3}{*}{$30 m$} & \multirow[b]{3}{*}{ - } & \multirow[b]{3}{*}{ - } & - & Aleatória & 0,25 & \multirow{3}{*}{$\begin{array}{l}\text { MELO } \\
(2009)\end{array}$} \\
\hline & & $\begin{array}{c}\text { Fragmentos } \\
\text { florestais naturais } \\
\text { (Ipucas) }\end{array}$ & & & & & - & Agregada & 16,07 & \\
\hline & & $\begin{array}{c}\text { Ecótono Cerrado/ } \\
\text { F. Amazônica }\end{array}$ & - & & & & - & Agregada & 3,51 & \\
\hline
\end{tabular}


Para SCHIAVINI (1992), em uma das duas áreas de seu estudo com três ambientes distintos (meio, dique e borda), descreveu a predominância de indivíduos com maiores alturas $(8,1$ a $10 \mathrm{~m})$, localizados na segunda área (meio da mata) e no dique, caracterizada por áreas elevadas entre as depressões e o ribeirão, indicando que os indivíduos da borda possivelmente sofram coma transição da mata de galeria e vegetação campestre, pois apresentaram as menores classes de tamanho $(50 \mathrm{~cm}$ a $6 \mathrm{~m})$. As classes de diâmetro pouco variaram (5 a $60 \mathrm{~mm}$ ) entre as três áreas, mas houve uma maior ocorrência de maiores diâmetros na área do meio devido a esta ser uma área de acúmulo de enchentes sazonais reforçando, portanto que, mesmo lentamente e com condições desfavoráveis os indivíduos estão em constante processo de regeneração.

Nestas áreas, a saturação ou completo alagamento do solo pela água superficial ou subterrânea seleciona organismos com adaptações para viver em solos mal drenados (JUNK \& DA SILVA, 1999; RIBEIRO \& WALTER, 2008), além daqueles que estão adaptados para o ambiente sem saturação. MARQUES (1994) no município de Brotas - SP relatou as distribuições em classes de altura e diâmetro dessa espécie apresentando uma curva do tipo "J invertido", evidenciando a estabilidade da população e a sua capacidade de regeneração, mesmo a área apresentando um considerável grau de perturbação antrópica. Para esse estudo, quando testado a distribuição dos valores de CAP por transecto não foram encontradas diferenças significativas ( $F$ valor $=0,767 ; p=0,386)$, conforme pode ser visto na figura 3 , confirmando que mesmo contendo algumas diferenças nos transectos, o comportamento dos indivíduos segue padrões estruturais semelhantes.

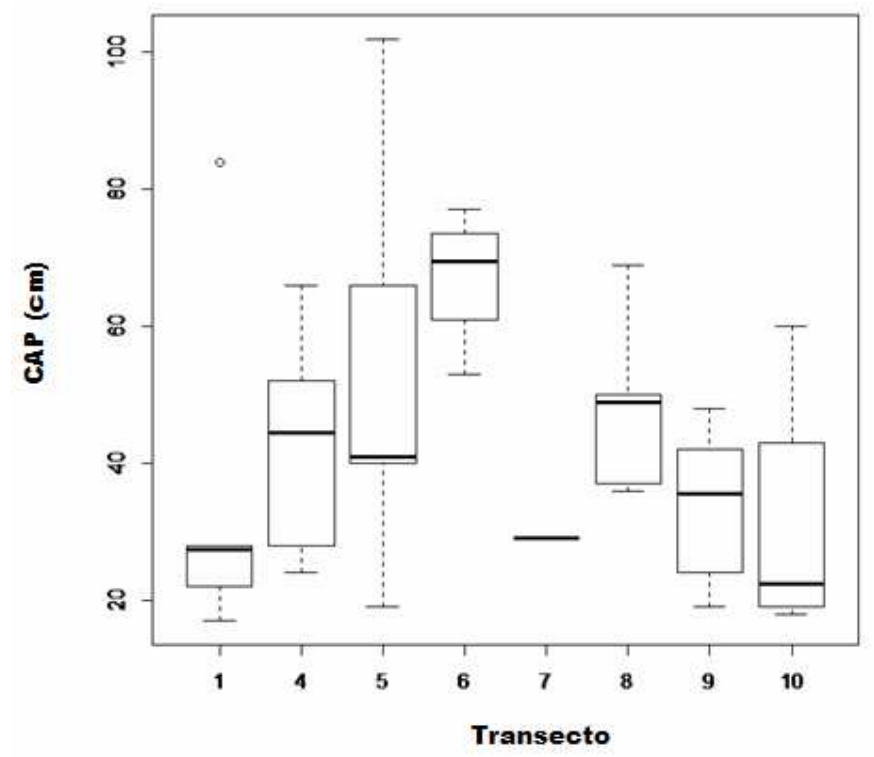

FIGURA 3. Reprodução gráfica em boxplot dos valores de CAP/Transecto na área do Rio Guaporé, teste $\mathrm{F}$ e valor de significância de P (ANOVA).

A curva hipsométrica indica uma relação positiva entre altura e circunferência (CAP) em uma determinada data (SCHIMIDT, 2007) com taxas constantes de mortalidade e recrutamento (HETT \&LOUCKS, 1976; SILVERTOWN,1987, AGREN \& ZACKRISSON, 1990). De acordo com a distribuição dos indivíduos o resultado da relação hipsométrica entre altura e CAP (Figura 4) demonstra que, a maioria dos 
indivíduos estão incluídos na altura entre quatro e oito metros e circunferência inferior a $50 \mathrm{~cm}$. $O \mathrm{R}^{2}$ dessa relação resultou em valores baixos para a área $(0,566)$ evidenciando baixa correlação.

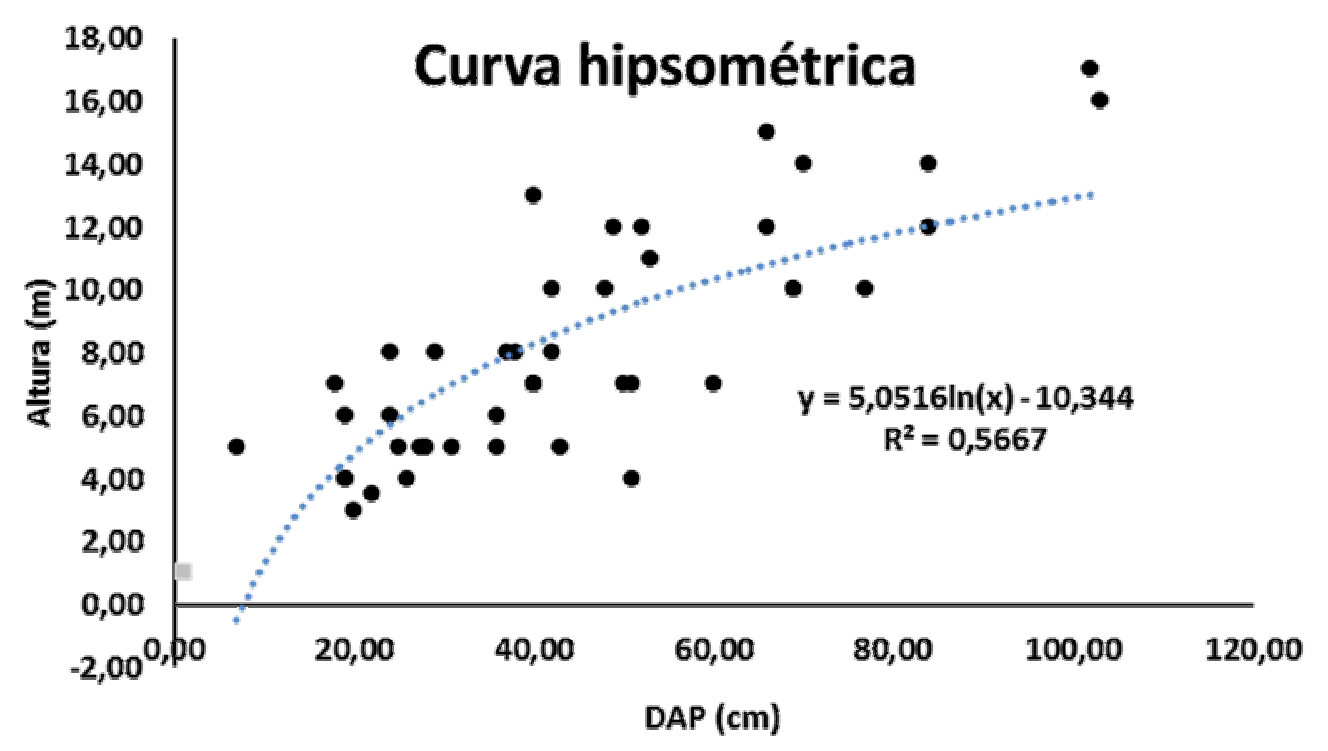

FIGURA 4. Relação entre altura e circunferência (CAP) dos indivíduos de uma população de Calophyllum brasiliense Cambess. em uma área de mata ciliar no Rio Guaporé,situada no Vale do Alto Guaporé, município de Vila Bela da Santíssima Trindade, Mato Grosso.

O índice de agregação de Morisita é pouco influenciado pelo tamanho da unidade amostral, apresentando excelentes qualidades de detecção (NASCIMENTO, 2001). Com base no Id conclui-se que os indivíduos estão distribuídos aleatoriamente dentro das parcelas, com valor resultante do Imst $(0,49)$, diferente do encontrado por MARQUES \& JOLY (2000), que foi agregada para todas as classes de tamanhos.

Para estudos realizados no Estado de Tocantins por MELO (2009), na Floresta Amazônica, em uma das três áreas analisadas, a distribuição espacial de indivíduos de $C$. brasiliense ocorreu de forma aleatória $(P=0,25)$, onde os indivíduos eram adultos com cerca de $30 \mathrm{~m}$ de altura e a área encontrava-se em um ambiente com pouca interferência antrópica, corroborando com esse estudo. A segunda área deste mesmo estudo se encontra em um ambiente em formação de Ipuca, que são fragmentos de floresta alagável, com poucos indivíduos adultos e a maioria jovem, a distribuição ocorreu de forma agregada $(P=16,07)$. Com índice de agregação baixo em relação a área dois e apresentando indivíduos de $C$. brasiliense em menor número, na área três a distribuição também ocorreu de forma agregada $(P=3,51)$, com médias semelhantes entre jovens e adultos.

NASCIMENTO (2001) atesta que, para o padrão aleatório, as espécies vegetais apresentam grande porte, expressando menores valores das médias no número de indivíduos e baixos valores da Razão (geralmente menores que um), pois por serem espécies que apresentam maior resistência em ambientes úmidos, aumentado assim a longevidade, podem aproximar-se de sua distribuição natural na 
floresta. Devido a tais fatores, torna-se necessária a preservação e conservação das áreas de ocorrência natural dessa espécie, contribuindo também para a manutenção de outras espécies, que apesar de não serem consideradas especialistas nesse tipo de habitat, compartilham o espaço e os recursos disponíveis no local.

\section{CONCLUSÃO}

Calophyllum brasiliense apresentou elevada abundância, em comparação aos outros estudos. Nas classes de altura e diâmetro, predominam indivíduos nas menores classes indicando que a espécie se encontra em regeneração apresentando curva do tipo $\mathrm{J}$ invertido. Conclui-se ainda que a distribuição espacial da espécie observando os resultados estatísticos é aleatória para o local. Este padrão de distribuição pode ser explicado devido às condições de saturação do ambiente ou à presença de ação antrópica no local.

\section{REFERÊNCIAS}

AB'SABER, A. N. Domínios morfoclimáticos e províncias fitogeográficas do Brasil. Orientação, v. 3, p. 45-48, 1967.

AGREN, J.; ZACKRISSON, O. Age and Size Structure of Pinussylvestris Populations on Mires in Central and Northern Sweden. Journal of Ecology, v. 78, n. 4 pp. 10491062, 1990.

ARIEIRA, J.; DA CUNHA, C. N.; Fitossociologia de uma floresta inundável monodominante de Vochysia divergens Pohl (Vochysiaceae), no Pantanal Norte, MT, Brasil. Acta Botanica Brasilica, v. 20, p. 569-580, 2006.

ASSUNÇÃO, S. L.; FELFILI, J. M. Fitossociologia de um fragmento de cerrado sensu stricto na APA do Paranoá, DF, Brasil. Acta Botanica Brasilica, v. 18 p. 903-909, 2004.

BELLUTA, I. Caracterização fitossociológica da vegetação ripária e qualidade da água do córrego do Cintra (Botucatu - SP) em função da ação antrópica. Tese de doutorado - Programa de pós-graduação em agronomia "Energia na Agricultura", Faculdade de Ciências Agronômicas da UNESP, Botucatu - SP, 2012.

BONINI, E.E. ; BONINI, S.E. Estatística: teoria e exercícios. Nobel. 1972.

BRASIL, M. M. E. Projeto RADAM: folha SD-20 Guaporé. Levantamento de Recursos Naturais, DNPM/CPRM, v.19, p. 369, 1979.

BRASIL - MINISTÉRIO DO MEIO AMBIENTE - Secretaria de Recursos Hídricos. Plano Nacional de Recursos Hídricos. Panorama e estado dos recursos hídricos do Brasil: Volume 1 / Brasília: MMA, 2006.

DEVIDE, A. C. P; CASTRO, C. M. de; PEREIRA, M. G.; RIBEIRO, R. de L. D.; ABBOUD, A. C. de S.; ASSUMPÇÃO, P. A. de; MAGALHÃES, P. M. de. Desenvolvimento do Guanandi (Calophyllum braziliense) em dois ambientes visando à conversão agroflorestal. Anais... VIIICBSAF, Belém, PA: SBSAF: Embrapa Amazônia Oriental: UFRA: CEPLAC: EMATER: ICRAF, p. 7, 2011. 
FACHIM, E.; GUARIM, V. L. M.S. Conservação da biodiversidade: espécies da flora de Mato Grosso. Acta Botânica Brasílica, Mato Grosso, v. 9, n. 2, p. 281-287, outubro, 1995.

FOSCHIERA, A. A.; MIORIM, V. M. F. Globalização e movimentos sociais no campo: $\mathrm{O}$ movimento união dos lavradores do Vale do Guaporé (MT). Fragmentos de Cultura. Goiânia, v. 16, n. 11/12, p. 885-906, 2006.

GAMA, J. R. V.; BOTELHO, S. A.; BENTES-GAMA, M. M.; SCOLFORO, J. R. S.; Estrutura e potencial futuro de utilização da regeneração natural de floresta de várzea alta no município de Afuá, estado do Pará. Ciência Florestal, Santa Maria, v. 13, n. 2, p. 71-82, 2003.

GUARIM NETO, G.; GUARIM, V. L. M. M. S.; NASCIMENTO, N. P. O. Etnobotânica no Pantanal: $O$ saber botânico tradicional pantaneiro. Flovet, n. 2, p. 1-68. Dezembro, 2010.2 Disponível em < http://periodicoscientificos.ufmt.br/index.php/flovet/article/view/644/571> .

HAIRSTON, N. G., HILL, R. AND RITTE, U.; The interpretation of aggregation patterns. In: PATIL, G. P.; PIELOU, E.C.; WATERS, W. E. Statistical Ecology. The Pennsylvania State University. Department of Statistics. University Park, p. 30, 1971.

HETT, J. M.; LOUCKS, O. L.Age Structure Models of Balsam Fir and Eastern Hemlock. Journal of Ecology, v. 64, n. 3, pp. 1029-1044, Nov., 1976.

INSTITUTO BRASILEIRO DE GEOGRAFIA E ESTATÍSTICA - IBGE. Área Territorial Brasileira. Disponível em http://www.ibge.gov.br/home/geociencias/areaterritorial/principal.shtm. Acesso em 02 de abril de 2015.

JANZEN, D. H. Herbivores and the number of tree species in tropical forests .American Naturalist. Chicago, v. 104, n. 940, p. 501-528, 1970.

JUNK, W. J. The flood pulse concept in river floodplain systems, p. 110-127. In D. P.Dodge [ed.] Proceedings of the International Large River Symposium. 1989.

JUNK, W. J.; DA SILVA, C. J. O conceito do pulso de inundação e suas implicações para o Pantanal de Mato Grosso. In: II Simpósio sobre Recursos Naturais e Socioeconômicos do Pantanal: Manejo e conservação, p. 17-28, 1999.

JUNK, W. J.; NUNES DA CUNHA, C.; SILVA, C. J. da; WANTZEN, K. M. The Pantanal: a large South American wetland and its position in limnológica theory. In: JUNK, W. J.; SILVA, C. J. da; NUNES DA CUNHA, C.; WANTZEN, K. M. The Pantanal: ecology, biodiversity and sustainable management of a large neotropical seasonal wetland. Sofia-Moscow: Pensoft Publishers, p. 26-46, 2011.

KOEPPEN, W. Climatologia; México, Fundo de Cultura Econômica, p. 466, 1948. 
KREBS, C. J. Ecological Methodology. Addison Wesley Educational Publishers, Menlo Park, p. 53, 1999.

LORENZI, H. Árvores brasileiras: manual de identificação e cultivo de plantas arbóreas nativas do Brasil. Nova Odessa: Plantarum, p. 382, 1992.

MAITELLI, G. T. Interações atmosfera-superfície. Geografia de Mato Grosso: território, sociedade e ambiente, p. 239-249, 2005.

MARQUES, M. C. M. Estudos autoecológicos do guanandi (Calophyllum brasiliense Camb. Clusiaceae) em uma mata ciliar do município de Brotas, SP. Dissertação de mestrado, Universidade Estadual de Campinas, Campinas. 1994.

MARQUES, M. C. M.; JOLY, C. A. Estrutura e dinâmica de uma população de Calophyllum brasiliense Camb. em floresta higrófila do sudeste do Brasil. Revista Brasileira de Botânica, v. 23, n. 1, p. 107-112, 2000.

MELACK, J. M.; HESS, L. L. "Remote sensing of the distribution and extent of wetlands In: the Amazon Basin" in: Amazonian floodplain forests: ecophysiology, biodiversity and sustainable management. Org. porJUNK, W. J. et al. (ed). Springer Verlag, New York, p. 43-60, 2010.

MELO, W. G da P. Distribuição biogeográfica de leveduras associadas a exsudados de Calophyllum brasiliense (Clusiaceae), em áreas de ecótono da planície do Araguaia - TO. . Dissertação (Mestrado) - Programa de Pós Graduação em Ciências do Ambiente, Universidade Federal do Tocantins/UFT. Palmas - TO. 2009.

MORISITA, M. Measuring of the dispersion of individuals and analysis of the distributional patterns. Mem. Fac. Sci. Kyushu University, v. 2, p. 215-235, 1959.

MORISITA, M. Id-index, a measure of dispersion of individuals.Researches on Population Ecology, v.4, p. 1-7, 1962.

MORO, M. F.; MARTINS F. R. Métodos de levantamento do componente arbóreoarbustivo. In: FELFILI , J. M.; EISENLOHR, P. V.; MELO, M. M. R. F.; ANDRADE, L. A.; NETO, J. A. M. (eds). Fitossociologia no Brasil: Métodos e estudos de casos. v. 1, Viçosa, MG: Ed. UFV, p. 174-212, 2011.

MOSS, G.; MOSS, M. Projeto Brasil das águas: Sete Rios, Guaporé. Brasília, 2007. Disponível em: < http://brasildasaguas.com.br/wpcontent/uploads/sites/4/2013/05/Rio-Guapore\%C2\%81 Relatorio.pdf> . Acesso em: 20 de Maio de 2013.

NASCIMENTO A. R. T.; LONGHI, S. J.; BRENA, D. A. Estrutura e padrões de distribuição espacial de espécies arbóreas em uma amostra de floresta ombrófila mista em Nova Prata, RS. Ciência Florestal, Santa Maria, v. 11, n.1, p.105-119, 2001. 
OLIVEIRA, V. C.; JOLY, C. A. Flooding tolerance of Calophyllum braziliense Camb. (Clusiaceae): morphological, physiological and growth responses. Trees, v.24, 185193. 2010.

PAULA, A. de; SOARES, J. J. Estrutura horizontal de um trecho de floresta ombrófila densa das terras baixas na reserva biológica de sooretama, Linhares, ES. Floresta, Curitiba, v. 41, n. 2, p. 321-334, abr./jun., 2011.

PEREIRA, L. A.; PEREIRA, M. C. T. Bacia Hidrográfica e Sua Relação Com o Ecossistema Ripário. In: ALVARES, I. A.; OLIVEIRA, A. R.; Anais do I Workshop sobre recuperação de áreas degradadas de mata ciliar no Semiárido Petrolina: Embrapa Semiárido, 98 p.: il. (Embrapa Semiárido. Documentos, 234), 2010.

PIERANGELI, M. A. P.; SADAYUKI, E.; ERGUCHI, R. F. R.;FERREIRA COSTA, D. F. V. Teores de As, $\mathrm{Pb}, \mathrm{Cd}$ e $\mathrm{Hg}$ e fertilidade de solos da região do Vale do Alto Guaporé , sudoeste do estado de Mato Grosso. Acta Amazônica, v. 39, p. 61 - 70, 2009.

REIS, C. A. F; DE SOUZA, A. M.; MENDONÇA, E. G.; GONÇALVEZ, F. R.; MELO, R. M. G.; DE CARVALHO, D. Diversidade e estrutura genética espacial de Calophyllum brasiliense camb. (Clusiaceae) em uma floresta paludosa. Revista Árvore, Viçosa-MG, v. 33, n. 2, p. 265-275, 2009.

RIBEIRO, J.F.; WALTER, B. M. T. As principais fitofisionomias do bioma Cerrado. In: SANO, S. M.; ALMEIDA, S. P.; RIBEIRO, J. F. (eds). Cerrado: ecologia e flora. Brasília/DF: Embrapa Cerrados/Embrapa Informação Tecnológica, v.1, p.151 - 212, 2008.

SARMENTO, B. M.; CORRÊA, B. S.; LOURES, L.; DE MOURA, A. S. Avaliação do desenvolvimento de mudas nativas em uma área paludosa, no município de Inconfidentes, MG. Revista Agrogeoambiental, Pouso Alegre, v. 5, n. 2, caderno I, p. 63-82, 2013.

SCHIAVINI, I. Estrutura das comunidades arbóreas de mata de galeria da Estação Ecológica do Panga, Uberlândia - MG. Tese de doutorado - Programa de pós - graduação em Ciências (Ecologia). Instituto de Biologia da Universidade estadual de Campinas, 1992.

SILVERTOWN, J. Ecologicalstability: a test case. American Naturalist, p. 807-810, 1987.

WEBB, L.J., TRACEY, J.G.; WILLIAMS, W.T. Regeneration and pattern in the subtropical rain for-est. Journal of Ecology, n. 3, p. 675-695, 1972. 